\title{
Using 6 Sigma to Improve Outcomes of Higher Education Institutes
}

\author{
Fuad M. Alkoot
}

\begin{abstract}
Higher education institutes face many challenges that require robust and scientific solutions. Six-sigma process improvement methodology is an example of a scientific method that we aim to use to improve the outcome of an educational institute. Six sigma is implemented successfully in the services and manufacturing sectors but rarely in education. Our aim is to show that six-sigma can be used in an educational institute of higher education and to show how it can be implemented. We use this scientific method for process control in order to improve the student outcome. We implement the first two phases of the six sigma method. In the first phase we state the problem and in the second phase we collect data from real cases at the Higher Institute of Telecommunication and Navigation. The obtained results will lead to an improvement of skills and knowledge attained by graduates of the institute. Preliminary results show that it diagnosis if problems exist and sets the path for finding causes that we must deal with to obtain improvements.
\end{abstract}

Index Terms-Quality outcome in education, six sigma, process improvement in education, higher education management.

\section{INTRODUCTION}

To tackle challenges faced by educational institutions state of the art methods are needed to improve the performance of all operations, managerial and educational. In [1] we used machine-learning tools to predict student outcome in order to find causes leading to declined student performance. Machine learning tools are useful on data with known inputs, or causes of an output. However, a more comprehensive method that can investigate the unknown causes and find solutions is required. One such method that has been widely used by large and successful industrial and services companies is the six-sigma process control. Deming [2] states that the education system can be improved using the same principles that are used to improve processes in other industries. Both academic and nonacademic processes must and can be improved using six sigma. Norma Simons [3] indicates 7 benefits of six sigma in higher education. We outline the most important ones as: meet accreditation requirements, provide a template for problem solving, help establish measures, make processes visible, obtain info on voice of customer, identify and reduce hidden costs. According to [4]-[6] the six sigma approaches are still emerging in the university setting. Author in [7] surveys the literature between 2000 and 2016 on the use of six sigma in education and concludes that there has not been any conclusive findings with regard to the use of six sigma and

Manuscript received May 23, 2018; revised August 2, 2018.

Fuad M. Alkoot is with HITN-PAAET, Kuwait (e-mail: fm.alkoot@paaet.edu.kw,f_alkoot@yahoo.com). lean six sigma in university environments. Immense challenges hinder their successful development. They find that US and UK universities are most committed to their implementation while in the Middle Eastern only Saudi Arabia seems to be involved. Targets are mostly primary processes associated with teaching and supporting processes associated with financial and building maintenance. In teaching targets were processes related to improvement of course content and program improvement. However, very few cost/benefit analysis results are documented leading to the conclusion that more work is needed before making claims about what the six sigma method can achieve in academia. After surveying 110 papers published between 2010 to 2016, on the use of six sigma in education, Sylvie Nadeau [7] conclude that this approach is quite novel in academia. The few conclusive results available do not provide a definite answer to whether six sigma will be enough to sustain advantages over the long run. The principle obstacle to the broad use of six sigma is encountered at the step of defining the client and added value.Therefore, we will contribute to the scientific community by experimenting with the use of six sigma in academia and sharing our findings in relation to the feasibility and benefits of using six sigma in education.

We aim to use six sigma to improve the outcome of PAAET, namely the skilled student. Six sigma is a process improvement methodology that is commonly used in the manufacturing and services industries where it has shown a high success rate. However, it is rarely used in the education industry. Therefore, the aim of this project is twofold; to investigate causes of degraded outcome at HITN using six sigma, and to find out how effective is six sigma as an improvement tool in the education sector. In this paper we show how to implement six sigma in an educational institute and the partial results obtained from this implementation.

\section{BACKGROUND ON SIX SIGMA}

Six Sigma [8] is a highly disciplined process that helps us focus on developing and delivering near-perfect products and services. It aims to eliminate waste and inefficiency, thereby increasing customer satisfaction by delivering what the customer is expecting. It is a data driven methodology, and requires accurate data collection for the processes being analyzed. Six Sigma is a business-driven, multi-dimensional structured approach for:

1) Improving Processes

2) Lowering Defects

3) Reducing process variability

4) Reducing costs

5) Increasing customer satisfaction 
6) Increased profits

Engineer Bill Smith invented Six Sigma while at Motorola in the early 1980s, in response to achieving 10X reduction in product-failure levels in 5 years. A common definition of sigma that has also been mentioned in [8] is as follows: "The word Sigma is a statistical term that measures how far a given process deviates from perfection".

The six sigma methodology measures the number of defects in a process and aims to eliminate the defects as much as possible. A process has a six sigma rating when its defects is reduced to 3.4 defects per one million opportunities for defects. This amounts to a process that produces items without defects $99.9997 \%$ of the time. There are two main six sigma methodologies which are used in two scenarios; when creating a new process or when improving an existing process. These two methodologies are define by [8] as:

"DMADV: It refers to a data-driven quality strategy for designing products \& processes. This methodology is used to create new product designs or process designs in such a way that it results in a more predictable, mature and defect free performance. This does not apply to our intended project.

DMAIC: It refers to a data-driven quality strategy for improving processes. This methodology is used to improve an existing business process. It consists of five steps; Define, Measure, Analyze, Improve and Control.

The DMAIC methodology consists of the following five steps:

1) Define: We find and focus on a single problem in the process and outline the project goals to solve the problems.

2) Measure: Data are collected from the process that is producing the problem.

3) Analyze: Conduct an analysis of the data to determine root causes of the problem.

4) Improve: The process is improved by finding solutions to prevent future problems.

5) Control: The implementation of the improved procedures is monitored to keep the process on the new course.

\section{EXPERIMENTAL METHODOLOGY}

In this paper, the five DMAIC stages of six-sigma were applied in sequence. We obtained the data needed for the analysis from the HITN database.

We start with the Define phase and undertake four major tasks as recommended by Pyzdek and Keller [8], which we quote as: "Project team Formation, Document Customers Core Business Processes, Develop a Project Charter, Develop the SIPOC process map."

\section{A. Project Team Formation}

Perform two activities; Determine who needs to be on the team and What roles will each person perform?"

\section{B. Document Customers Core Business Processes}

Every project has customers. A customer is the recipient of the product or service of the process, targeted for improvement. Every customer has one or multiple needs from his or her supplier. For each need provided for, there are requirements for the need. The requirements are the characteristics of the need that determine whether the customer is happy with the product or service provided. So, document customer needs and related requirements. A set of business processes is documented. These processes will be executed to meet customer's requirements and to resolve their Critical to Quality issues."

\section{Develop a Project Charter}

This is a document that names the project, summarizes the project by explaining the business case in a brief statement, and lists the project scope and goals. "

\section{Develop the SIPOC Process Map}

A process is defined as a series of steps and activities that take inputs, add value, and produce an output. SIPOC is a process map that identifies all the following elements of a project: Suppliers, Input, Process, Output, Customers. The SIPOC process map is essential for identifying the way processes occur currently, and how those processes should be modified and improved throughout the remaining phases of DMAIC."

During the second phase, the Measure Phase, we measure the performance of the process at different stages and locations affecting the output. Therefore, at this stage we make a plan of how and what data to collect. Then the data is collected and then evaluated. One commonly used tool at this stage is the Failure Mode and Effects Analysis - FMEA.

At the third stage, the analysis phase, we analyze the causes of defects and measure the defects. Among the many causes are causes due to the process, procedure or resources. Solutions to causes of defects are investigated at the end of this stage.

The objective of Improve Phase is to identify improvement breakthroughs, identify high gain alternatives, select preferred approach, design the future state, determine the new Sigma level, perform cost/benefit analysis, design dashboards/ scorecards, and create a preliminary implementation plan.

At the last stage, the control phase, we ensure that the newly implemented and improved processes continue to work well. If the output deviates from the newly set target level, an alarm is raised. Then, the process is checked before the output is affected or defects are produced. This ensures to maintain the quality at the desired sigma performance level. Mainly the procedures where defects are most likely to occur are closely monitered using sepcially designed control guidelines.

For each process, we need to define and measure process metrics. We are investigating the process that produces skilled students. Therefore, our measurement metric are the quiz, midterm and final exam results. Measurement system analysis is used to determine if midterm \& final exams are accurate representation of students gained knowledge and skills.

Process baseline definition: we will establish a process baseline which provides a measure of process performance before improvement. Find mean and standard deviation after checking the following: (i.e. SPC provides estimates of mean and standard deviation (process location and variation) to compare with existing requirements.):

We also will use SPC to define process baseline. If stable then process capability and sigma level estimates can be used 
to quantify the performance of the process relative to requirements. If not stable then causes of variation need to be found. If found and removed then stable baseline process is established and compared to requirement. If not found then we need to use DOE in the analyze phase to find causes of instability.

Process baseline also determines if project charter is right in the need for correction. It also helps identify critical CTQs, which need to be addressed and it also helps determine the next strategy. Erratic and unstable performance needs a different strategy than if performance is consistently poor. Process baseline also provides information on magnitude of improvement and savings.

We focus on a certain class or subject and consider it as an indicator. This can be repeated individually for each class. (In this paper the words class and subject indicate the same thing and both can be used). For this certain class of many students we have a group at a session of a year.

If the chart displays average per group then the averages are broken down for separate years and sessions. If we display averages per session then this average is for many groups at a session of a year.

\section{Process Baseline AND DAta SeT}

We establish a process baseline which provides a measure of process performance before improvement. SPC provides estimates of mean and standard deviation (process location and variation) to compare with existing requirements. Therefore, we find mean and standard deviation after checking the following:

Use SPC to define process baseline. If stable then process capability and sigma level estimates can be used to quantify the performance of the process relative to requirements. If not stable then causes of variation need to be found. If found and removed then stable baseline process is established and compared to requirement. If not found then we need to use DOE in the analyze phase to find causes of instability.

Process baseline also determines if project charter is right in the need for correction. It also helps identify critical CTQs, which need to be addressed and it also helps determine the next strategy. Erratic and unstable performance needs a different strategy than if performance is consistently poor. Process baseline also provides information on magnitude of improvement and savings.

We focus on a certain class or subject and consider it as an indicator. This can be repeated individually for each class. (In this report the words class and subject indicate the same thing and both can be used). Each class includes many groups of students. Each class is also repeated for two sessions of a year, for many years.

If the chart displays average per group then the averages are broken down for separate years and sessions. If we display averages per session then this average is for many groups at a session of a year.

The data of student performance results are continuous where we have 44 groups with larger than 10 group size. For each group we have 10 classes or subjects given code numbers 228, 229, 230, 233, 234, 277, 278, 279, 280, 282. For each class we have many students that exceed 10 per group. For each student we have midterm and final exam results. Final exam results are a number between 0 and 100. A group may include all male or all female students.

\section{RESUlTS}

Given the data for the 44 groups we will proceed with plotting average \& standard deviation control charts. As shown in Fig 1 and Fig 2, for classes 279 and 282.

$$
\begin{gathered}
A_{\text {subgroup average }}=\sum \frac{\text { subgroup measurements }}{\text { subgroup size }} \\
S_{\text {subgroup standard deviation }}=\sqrt{\frac{\sum_{i=1}^{n}\left(x_{i}-A v e\right)^{2}}{n-1}}
\end{gathered}
$$

Control limit for standard deviation charts:

$$
\begin{aligned}
& S_{\text {ave }}=\frac{\sum S_{i}}{\text { number of subgroups }} \\
& \mathrm{LCL}=\mathrm{B} 3 \times S_{\text {ave }} \text { where B3 }=0.565 \\
& \mathrm{UCL}=\mathrm{B} 4 \times S_{\text {ave }} \text { where B4 }=1.435
\end{aligned}
$$

Control limit for average charts:

$$
\begin{aligned}
A_{\text {ave }} & =\frac{\sum \text { Ave }_{i}}{\text { number of subgroups }} \\
\mathrm{LCL} & =A_{\text {ave }}-\mathrm{A} 3 \times S_{\text {ave }} \text { where A3 }=0.606 \\
\mathrm{UCL} & =A_{\text {ave }}+\mathrm{A} 3 \times S_{\text {ave }}
\end{aligned}
$$

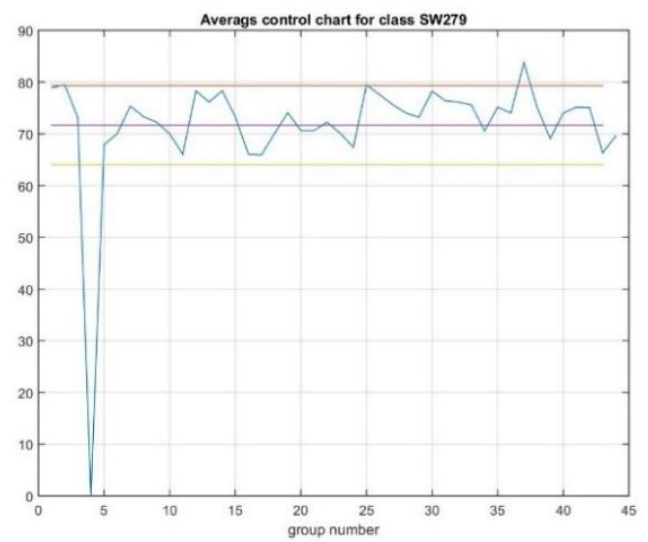

(a) Averages control chart for class SW279

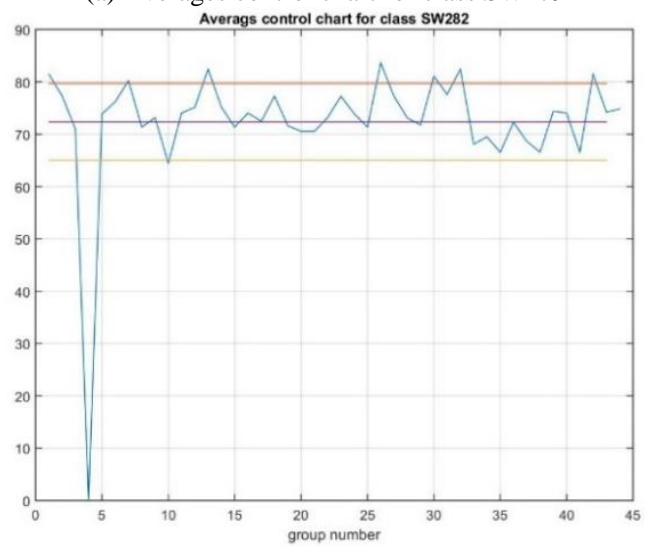

(b) Averages control chart for class SW282

Fig. 1. Averages control charts for two classes of EEM program at HITN. 
Plotting the values obtained from the equations above we get the chart graphs of Fig. 1 and Fig. 2.

We have to analyze the charts. Find special causes and fix them to obtain a controlled process and then you can find the capability index. Looking at the figures the plots can be partitioned into three zones [8]. Zone $\mathrm{A}$ is between 2 and 3 standard deviations. Zone $\mathrm{B}$ is between 1 and 2 standard deviations. Zone $\mathrm{C}$ is below 1 standard deviation.

Based on the zones and the trends of values on the curve we can determine special causes of variation when any of the following conditions occur:

- A single point on the curve is beyond any one of the control limits.

- 2 out of 3 consecutive points appear in Zone A.

- 4 out of 5 consecutive points appear in Zone B.

Other trends that require attention are:

- 9 successive on one side of the center line.

- 15 points in Zone $\mathrm{C}$ on one side. This indicates permanent change and requires precomputing control limits.

- 7 continued rise or fall points. Show a trend of gradual change.

- 14 Cycle or repeated alternating rise and fall indicate the cause is cyclic with repetitive effect.

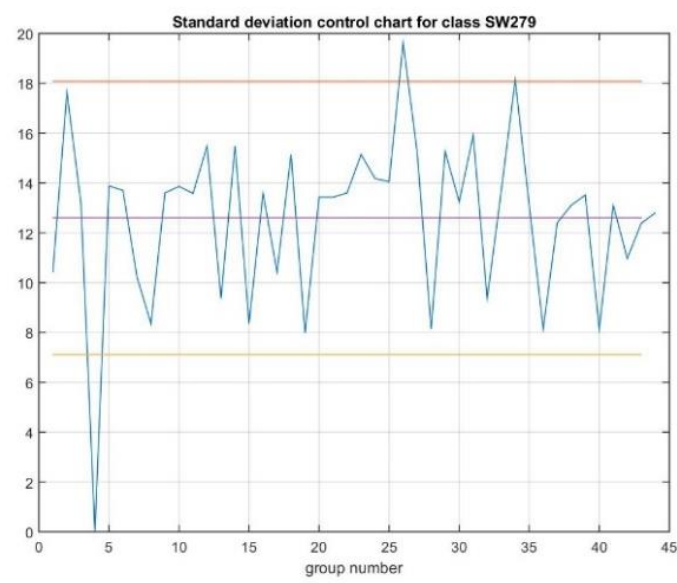

(a) Standard deviation control chart for class SW279

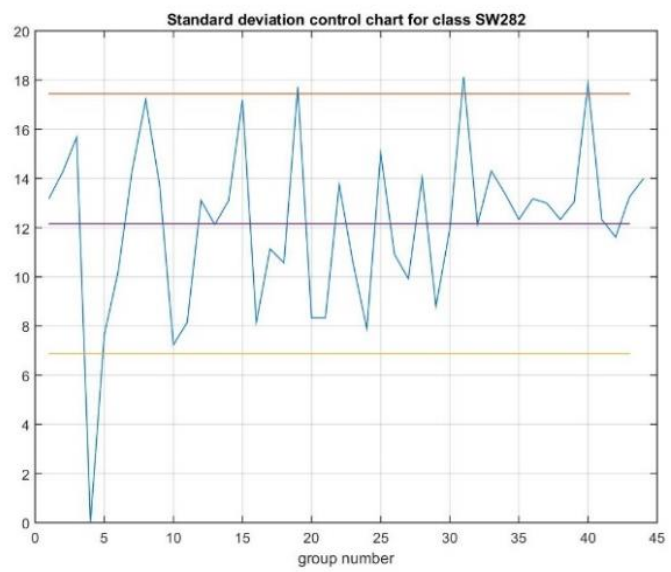

(b) Standard deviation control chart for class SW282

Fig. 2. Standard deviation control charts for two EEM-HITN classes.

Looking at the plots of average charts for the different subjects we don't see any of the trends mentioned here except for points falling outside the control limits. These indicate special causes that will be investigated. This indicates that our definition of the problem is correct and a problem exists.

One of the main procedures than must be implemented is the measurement system analysis to confirm that our measurement system is valid. Two main parameters of the measurement system that need to be checked before proceeding with the analysis of measurements are "repeatability" and "reproducibility".

Repeatability (precision) or Equipment variation is Variation in measurements. Range is between different readings of same part and same appraiser.

Reproducibility is Appraiser variation. Variation in average of measurements between appraisers.

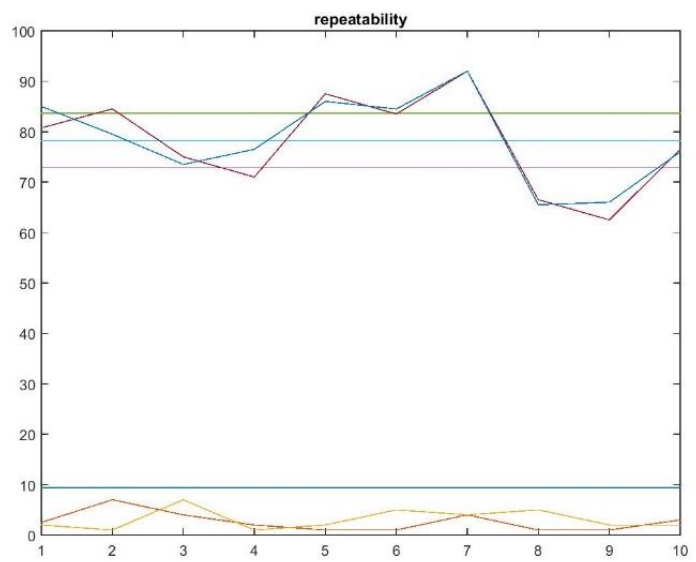

Fig. 3. Plots for range chart (lower curve) and for average chart (upper curve) for the measurement system repeatability test.

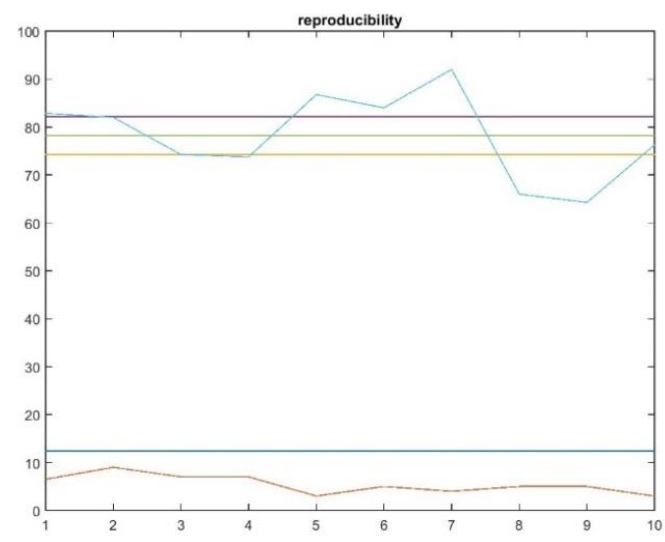

Fig. 4. Plots for range chart (lower curve) and for average chart (upper curve) for the measurement system reproducibility test.

A measurement system is reproducible when different appraisers produce consistent results. Therefore, we compare appraisers' average with that of other appraisers. We compared the measurements of two different teachers (i.e. appraisers) at two groups of students for the same subject.

Looking at figures 3 and 4 we find that our measurement system is both reproducible and repeatable. Next phase involves analysis of the data and plots of figures 1 and 2 to find causes of variation.

Future work will involve further analysis to find causes of problems. Furthermore, improvement actions will be recommended for implementation.

\section{CONCLUSION}

In this paper we propose using the six sigma process improvement method to improve the outcome at an 
educational establishment. Six sigma is used in the manufacturing and services sectors. However, it is not used in an educational institute to improve the outcome, i.e. the student.

We show how it is possible to implement 6 sigma in a real case scenario and present in this paper the results of implementing the first two phases of six sigma, namely the define and measure phases.

\section{REFERENCES}

[1] F. M. Alkoot, "Using classifiers to predict student outcome at higher institute of telecommunication," in Proc. 18th International conference on machine learning and Data Analysis, Tokyo, Japan, May 26-27 2016

[2] E. Deming, The New Economics: For Industry, Government, Education, MIT press, 2nd ed. 1993.

[3] N. Simons, "The business case for lean six sigma in higher education," ASQ Higher Education Brief, vol. 6, no. 3, May 2013.

[4] S. Albliwi et al., "Crtitical failure factores of lean six sigma: A systematic literature review," International journal of Quality and Reliability Management, vol. 31, pp. 1012-1030, 2014
[5] Z. Radnot and G. Bucci, "Analysis of lean implementation in UK business schools universities," A to Z Business Consultancy, p. 74, 2011.

[6] R. Raifsnider and D. Kurt, "Lean six sigma in higher education; applying proven methodologies to improve quality remove waste, and quantify opportunities in colleges and universities,"Xerox White Paper.

[7] S. Nadeau, "Lean, six sigma and lean six sigma in higher education: A review of experiences around the world," American Journal of Industrial and Business Management, vol. 7, no. 5, May 22, 2017.

[8] T. Pyzdek and P. Keller, Six Sigma Handbook, $4^{\text {th }}$ ed. McGrawHill, 2014.

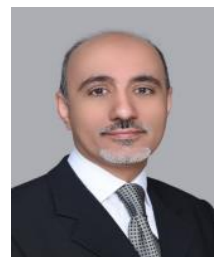

Fuad M. Alkoot obtained his $\mathrm{PhD}$ in electronic engineering from CVSSP - University of Surrey, UK in 2001. He obtained his M.Sc. And B.Sc. in electrical engineering from Rochester Institute of Technology, N.Y. and Fairleigh Dickenson University, N.J, USA, respectively. $\mathrm{He}$ is currently affiliated with HITN-PAAET, Kuwait, as an academic teaching staff. His main research interests are fusion methods, multiple classifier systems, biometric authentication methods, combiner methods and bioinformatics. 